\title{
Glossopharyngeal Schwannoma: Review of Five Cases and the Literature
}

\author{
T. A. Sweasey, M.D., S. R. Edelstein, and J. T. Hoff, M.D. \\ Section of Neurosurgery, University of Michigan Hospitals, Ann Arbor, Michigan
}

Sweasey TA, Edelstein SR, Hoff JT. Glossopharyngeal schwannoma: review of five cases and the literature. Surg Neurol 1991;35:127-30.

Glossopharyngeal schwannomas are rare tumors in spite of the fact that acoustic schwannomas account for $8 \%-10 \%$ of intracranial tumors. There have been 23 reported cases in the literature. This report of five cases is the largest series of these tumors. The presentation, radiological workup, operation, and long-term postoperative results will be presented, along with a review of the literature.

KEY WORDS: Glossopharyngeal; Schwannoma; Jugular foramen; Neurinoma; Cranial nerve tumor

Schwannomas arising from the glossopharyngeal nerve are exceedingly rare, and generally it is very difficult to determine the nerve of origin preoperatively. We have reviewed five cases of glossopharyngeal schwannomas that have been seen at this institution. Follow-up evaluations are available on each patient and average 4 years. The presentation, radiologic evaluation, operation, and long-term outcomes, along with a review of the literature, will be presented.

\section{Case Reports \\ Case 1}

A 24-year-old man, status post a motor vehicle accident in 1979, presented with a 14-month history of hearing loss. He had no complaints of loss of taste, difficulty with balance, or difficulty with swallowing. Neurological examination revealed decreased hearing on the right, but no dysmetria, diplopia, nystagmus, or ataxia. Preoperative computed tomography (CT) scan showed a 3-4$\mathrm{cm}$ diffusely enhancing mass lesion in the right cerebellopontine angle. Cerebral angiography showed posterior

Address reprint requests to: T. A. Sweasey, M.D., University of Michigan Hospitals, Section of Neurosurgery, 1500 East Medical Center Drive, Ann Arbor, Michigan 48109-0338.

Received April 30, 1990; accepted July 9, 1990. fossa vascular displacement, but no tumor blush. The patient underwent a right suboccipital craniectomy after ventriculostomy. Gross total resection of a ninth nerve schwannoma was accomplished. Postoperatively, there was mild right facial weakness with a diminished gag reflex on the right. There was no change in the patient's hearing loss. Follow-up evaluation 4 years after surgery demonstrated right-side hearing loss and decreased right palatal function. The CT scan showed no evidence for a recurrent tumor.

\section{Case 2}

A 32-year-old woman presented to an outside hospital with a 3-4-month history of decreased hearing in the left ear. Evaluation revealed a left cerebellopontine angle mass, which was felt to be an acoustic neuroma. A posterior fossa craniectomy was performed. The tumor was debulked and the pathologic diagnosis was schwannoma. Hearing improved postoperatively. Postoperative CT scan demonstrated residual tumor in the region of the jugular foramen, with enlargement of the foramen. Angiography showed vascular displacement, but was without tumor blush. Neurological examination at the time of referral showed only mild left palatal weakness and normal fifth, seventh, and eighth nerve function. She underwent a second posterior fossa craniectomy with resection of $90 \%$ of a ninth nerve schwannoma. Postoperatively, she had a partial vocal cord paralysis and difficulty with swallowing, which subsequently resolved. Over a 4-year course, she has had a recurrence of her tumor with both intracranial and extracranial components. At present she is intact neurologically and is being followed with clinical and radiological evaluation every 6 months.

\section{Case 3}

A 49-year-old woman was diagnosed and underwent partial resection of a left "acoustic neuroma" at age 19. She had presented with a 3-year history of decreased hearing on the left. In 1977, she underwent tumor debulking with resultant left fifth and seventh nerve palsies. 
In 1987, the patient underwent a third posterior fossa craniectomy with subtotal resection of a schwannoma of the ninth cranial nerve. Prior to this operation, she had developed truncal ataxia and atrophy of the left side of the tongue. Computed tomography and magnetic resonance imaging (MRI) demonstrated recurrent tumor, which involved the jugular foramen and extended into the cerebellopontine angle. Angiography revealed vascular displacement, but no tumor blush. Follow-up examination 2 years later showed left fifth, seventh, and ninth nerve palsies. Additionally, she had decreased hearing on the left. In 1989 MRI showed a $3 \times 3-\mathrm{cm}$ mass, enhanced with gadolinium, centered in the jugular forament, extending extracranially. With the patient being neurologically stable, this lesion is being followed clinically and radiographically.

\section{Case 4}

A 67-year-old woman presented in 1982 with decreased hearing on the right, double vision, and ataxia. Evaluation with CT scan revealed a mass that extended from the jugular foramen into the cerebellopontine angle. Cerebral angiography demonstrated vascular displacement, but was without tumor blush. She underwent a transmastoid retrolahyrinthine hiopsy of the mass, which revealed schwannoma. Postoperatively, she developed acute hydrocephalus with bilateral sixth nerve palsies and underwent placement of a ventriculoperitoneal shunt. Subsequently, she underwent a suboccipital craniectomy for tumor debulking of the ninth nerve schwannoma. Three months later she underwent a second operation via the same approach and a $90 \%$ resection of the tumor mass was achieved. She was treated with $5000 \mathrm{rads}$ to the tumor bed. Postoperatively, the patient had decreased palatal function on the right with a decreased gag reflex. The patient's gait was slightly ataxic. Her hearing loss persisted. Mild ninth nerve palsies secondary to the acute hydrocephalus also persisted. Follow up in 1989 showed no change in the patient's neurologic examination and C $\mathrm{C}$ 'scan showed no change in the slight residual tumor.

\section{Case 5}

This patient is a 35-year-old man who was evaluated at an outside facility for a hypoglossal palsy in 1980. Radiologic studies from that admission are unavailable, but operative records showed a glossopharyngeal schwannoma was subtotally resected via a suboccipital approach. In 1983, he had a recurrence of his tumor and underwent reoperation with subtotal resection. Postoperatively, he was treated with radiation therapy. In May 1985 , he once again had a recurrence of his tumor and a third suboccipital approach for tumor resection was performed. He has continued to have residual tumor on his postoperative MRIs; however, the tumor has been stable since 1985. His neurologic status at this time shows no hearing loss, but he does have hoarseness and leftward protrusion of the tongue.

\section{Discussion}

Schwannomas of the ninth cranial nerve are rare lesions. There are only 23 reported cases $[1-17]$. Often, tumors in the region of the ninth, 10 th, and 11 th cranial nerves are reported as jugular foramen schwannomas without a specific nerve of origin identifled. This series of five cases of ninth nerve schwannomas is the largest series to date. Additionally, long-term follow up is available for all five patients.

The glossopharyngcal ncrve is a mixed branchiomeric nerve that includes five different functional components. These are general visceral afferents (GVA), special visceral afferents (SVA), general somatic afferents (GSA), general visceral efferents (GVE), and special visceral efferents (SVE). The functions that these fibers subserve include taste on the posterior one third of the tongue (SVA), sensation behind the ear (GSA), sensation from the posterior upper pharynx, tonsils, the posterior one third of the tongue, and input from the carotid sinus (GVA), stylopharyngeus muscle and possibly some of the pharyngeal constrictors (GVE).

The glossopharyngeal nerve has its root in the dorsolateral sulcus of the medulla, just rostral to the vagus nerve. It proceeds ventrolaterally to the jugular foramen. The jugular foramen is divided into two sections by a fibrous, sometimes bony, septum. In the anteromedial compartment, cranial nerves IX, X, and XI, along with the inferior petrosal sinus, make their exit from the skull. The posterolateral compartment contains the sigmoid sinus as it forms the internal jugular vein and the posterior meningeal artery. One centimeter anterior and cephalad to the jugular foramen is the internal auditory canal.

Theoretically, the symptoms and signs of a tumor of the glossopharyngeal nerve should include dysfunction of all the fibers noted above, plus compressive symptoms of the $10 \mathrm{th}$ and $11 \mathrm{th}$ nerves. However, in our group of five patients and in the literature review, hearing loss was the most common finding in all of the patients. Twenty-five of 27 patients (one case report did not mention the hearing status) presented with symptoms of decreased hearing. This is even more remarkable as approximately $70 \%$ of patients with acoustic neuroma present with symptoms of hearing loss. Only two case reports listed a loss of taste as a presenting complaint. Hoarseness was present in eight patients and a decrease 


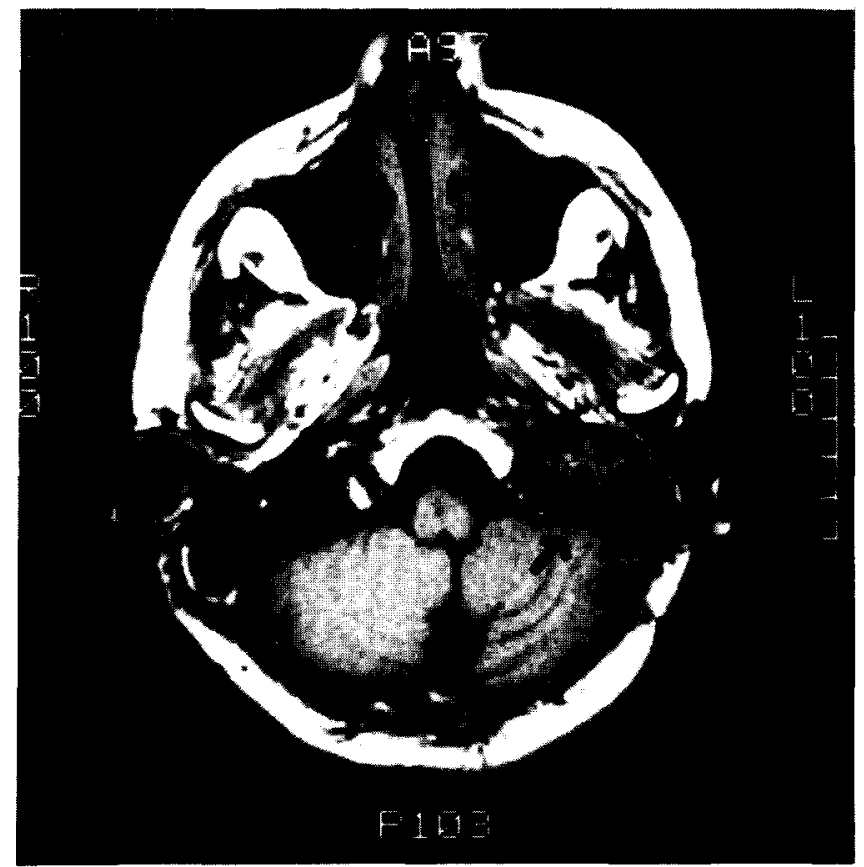

Figure 1. Axial T1-weighted MRI of a left ninth nerve schwannoma (arrow). The mass obliterates the internal jugular vein. This mass diffusely enbances with gadolinium.

in the gag reflex was found in six patients. Pain was present in only four patients but was not historically consistent with glossopharyngeal neuralgia. Most of the symptoms of glossopharyngeal dysfunction do not become apparent to patients until there is bilateral nerve involvement. Thus, these tumors can grow quite large prior to recognition. This also helps to explain the almost universal presentation of hearing loss. None of the patients described presented with a carotid sinus syndrome.

Patient population in all the cases in the literature had an age range of $15-67$ years, with 20 of 28 patients falling in the age range 20-49 years. The duration of symptoms was as short as 2 months and as long as 12 years. There is a male predominance in the literature of approximately $1.5: 1$ ( 17 men and 11 women). Our series of five consisted of two men and three women. There has been a suggestion of right-sided predominance of this tumor; however, the 28 tumors reviewed are equally divided between right and left [5].

Determining the glossopharyngeal nerve as the nerve of origin for the tumor is accomplished only at surgery. The preoperative clinical evaluation can lead to a suspected diagnosis of involvement of the cerebellopontine angle and possibly the jugular foramen, but is otherwise not specific. Radiologic workup, including CT, angiography, or plain $\mathrm{x}$-ray, can localize the lesion to the jugular foramen, but again, does not distinguish the particular

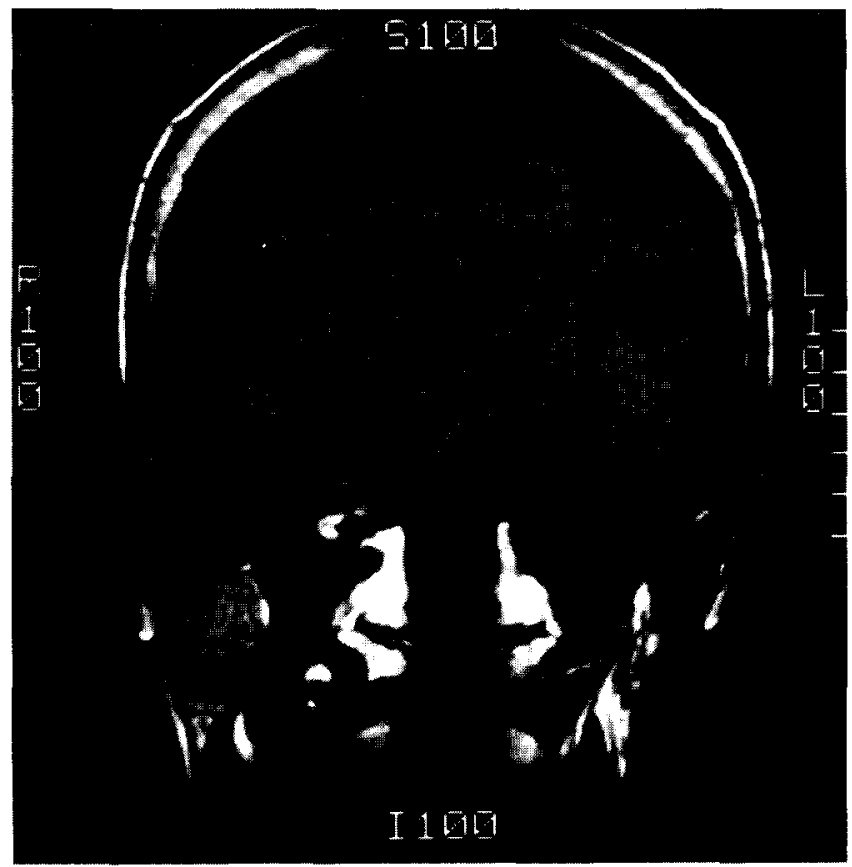

Figure 2. Coronal T1-weighted MRI from the same patient demonstrating the mass extending superiorly toward the brain stem (arrow).

nerve of origin of the tumor. Presentation of glossopharyngeal schwannomas on CT scan varies from a diffusely enhancing mass to ring enhancing to minimally enhancing. The bony margins around the tumor are generally smoothly eroded as opposed to the destructive changes associated with glomus jugular tumors. The middle ear usually is not involved and there is often a cystic component to the tumor. Angiography may show a slight tumor blush, but commonly only shows vascular displacement. Occasionally, the sigmoid sinus-internal jugular complex may be occluded. Magnetic resonance imaging will become an important procedure, as it gives excellent demonstration of the tumor (Figures 1 and 2). Enhancement of the tumor with gadolinium is similar to that on CT scan. The relationship of the tumor to the cerebellum and brain stem is well demonstrated. At the time of operation, the tumor may encase the ninth, 10th, and 11 th nerves, making the nerve of origin unidentifiable.

Based on the five cases noted in this report, total resection of these tumors without major disability, ie, ninth and 10th nerve palsies, is difficult at best. Only one patient of the five has had no evidence of tumor regrowth. These tumors tend to be slow growing, both initially and at the time of recurrence. The operative approach is dictated by the location of the tumor (intracranial, extracranial, or both). In the five cases reported herein, the approach for resection was a suboccipital craniectomy. The procedure is complicated by the prox- 
imity of cranial nerves $\mathrm{X}$ and $\mathrm{XI}$ to the neurinoma of cranial nerve IX. The goal is complete resection of tumor without an increase in neurologic deficit. As noted above, this is often not successful. Additionally, in some patients with large tumors, obstructive hydrocephalus may be present, requiring a ventriculostomy at the time of surgery. Case 4 illustrates that manipulation of the tumor during biopsy can precipitate acute obstructive hydrocephalus.

Neurologically, all five patients are stable in spite of significant tumor growth in several instances. Further operative intervention will be dictated by their neurologic status in relationship to the radiologic studies. Reoperation can be performed safely, as demonstrated in this report.

Two of the patients in this series were treated with radiation therapy. Radiation therapy is controversial with acoustic neuromas, and so the role of radiation for glossopharyngeal schwannomas is unclear. In those instances in which further resection of the tumor is not possible, then radiation therapy is certainly an option.

\section{Summary}

Five cases of glossopharyngeal neurinomas are added to 23 cases reviewed from the literature. Overall, it is noted that these tumors are more common in males with equal occurrence rates on either the right or the left. These tumors commonly occur in the third to fifth decades. The most common presenting symptom is hearing loss. From the long-term experience with our group of patients, it appears that complete resection is often not accomplished and many patients can have a recurrence of tumor. Since these tumors tend to grow slowly, patients can be followed clinically and radiographically if there is no progression of symptoms or signs. Radiation therapy can be used as an adjunct, but its overall role is not clear.

\section{References}

1. Andersen T, Ulsoe C, Overgaard J, Rignsted J. Intracranial glossopharyngeal schwannoma, a tumour imitating an acoustic schwannoma. J Laryngol Otol 1986;100:831-5.

2. Arenberg IK, McCreary HS. Neurilemoma of the jugular foramen. Laryngoscope 1971;81:541-7.

3. Brandenburg JH. Neurogenic tumors of the parapharyngeal space. Laryngoscope 1972;82:1292-305.

4. Call WH, Pulec JL. Neurilemoma of the jugular foramen transmastoid removal. Ann Otol 1978;87:313-7.

5. Claesen P, Plets C, Goffin J, Van den Bergh R, Baert A, Wilms $G$. The glossopharyngeal neurinoma. Clin Neurol Neurosurg 1989;91:65-9

6. Fink LH, Early CB, Bryan RN. Glossopharyngeal schwannomas. Surg Neurol 1978;9:239-45.

7. Hakuba A, Hashi K, Fujitani K, Ikuno $H$, Nakamura T, Inoue $Y$. Jugular foramen neurinomas. Surg Neurol 1979;11:83-94.

8. Healy JF, Goff WB, Bishop JW. Pathologic basis of computed tomographic and angiographic findings in a giant ninth nerve neuroma. Comput Radiol 1982;6:227-31.

9. House WF, Horn KL, Hitselberger WE. Schwannomas of the jugular foramen. Laryngoscope 1985;95:761-5.

10. Kaye AH, Hahn JF, Kinney SE, Hardy RW, Bay JW. Jugular foramen schwannomas. J Neurosurg 1984;60:1045-53.

11. Kelly DL, Britton BI I, Branch CL. Cooperative neuro-otologic management of acoustic neuromas and other cerebellopontine angle tumors. S Med J 1988;81:557-61.

12. Maniglia AJ, Chandler JR, Goodwin WJ, ParkerJC. Schwannomas of the parapharyngeal space and jugular foramen. Laryngoscope 1979;89:1405-14.

13. Martinez R, Vaquero J, Cabezudo J, Areitio E, Bravo G. Neurinomas of the jugular foramen in children. J Neurosurg 1981;54:693-5

14. Naunton RF, Proctor L, Elpern BS. The audiologic signs of ninth nerve neurinoma. Arch Otolaryngol 1968;87:20-5.

15. Pluchino F, Crivelli G, Vaghi MA. Intracranial neurinomas of the nerves of the jugular foramen. Acta Neurochir 1975;31:201-21.

16. Suzuki F, Handa J, Todo G. Intracranial glossopharyngeal neurinomas. Report of two cases with special emphasis on computed tomography and magnetic resonance imaging findings. Surg Neurol 1989;13:390-4.

17. Uchino A, Hasuo K, Fukui M, Matusushima T, Tamura S, Yasumori $\mathrm{K}$. Computed tomography of jugular foramen neurinomas. Neurol Med Chir (Tokyo) 1987;27:628-32. 